\title{
Multi-source Time Synchronisation in IEEE std 802.15.4-2015 TSCH Networks
}

\author{
Papadopoulos, Georgios Z.; Fafoutis, Xenofon; Thubert, Pascal
}

Published in:

Internet Technology Letters

Link to article, DOI:

10.1002/it|2.148

Publication date:

2020

Document Version

Peer reviewed version

Link back to DTU Orbit

Citation (APA):

Papadopoulos, G. Z., Fafoutis, X., \& Thubert, P. (2020). Multi-source Time Synchronisation in IEEE std 802.15.4-2015 TSCH Networks. Internet Technology Letters, 3(2), [e148]. https://doi.org/10.1002/itl2.148

\section{General rights}

Copyright and moral rights for the publications made accessible in the public portal are retained by the authors and/or other copyright owners and it is a condition of accessing publications that users recognise and abide by the legal requirements associated with these rights.

- Users may download and print one copy of any publication from the public portal for the purpose of private study or research.

- You may not further distribute the material or use it for any profit-making activity or commercial gain

- You may freely distribute the URL identifying the publication in the public portal

If you believe that this document breaches copyright please contact us providing details, and we will remove access to the work immediately and investigate your claim. 
DOI: $\mathrm{xxx} / \mathrm{xxxx}$

\title{
ARTICLE TYPE
}

\section{Multi-source Time Synchronisation in IEEE std 802.15.4-2015 TSCH Networks}

\author{
Georgios Z. Papadopoulos*1 | Xenofon Fafoutis ${ }^{2}$ | Pascal Thubert ${ }^{3}$
}

\author{
${ }^{1}$ IMT Atlantique, IRISA, France \\ ${ }^{2}$ Technical University of Denmark (DTU), \\ Denmark \\ ${ }^{3}$ Cisco Systems, France \\ Correspondence \\ *Georgios Z. Papadopoulos. Email: \\ georgios.papadopoulos@imt-atlantique.fr
}

\section{Present Address}

This is sample for present address text this is sample for present address text

\begin{abstract}
Summary
IEEE std 802.15.4-2015 Time Slotted Channel Hopping (TSCH) extends and replaces multiple proprietary industrial standards to support ultra-reliable and ultralow power operation in Industrial Internet of Things. Under TSCH, all devices must remain continuously synchronised with their time source neighbour. To do so, the nodes either periodically exchange control packets or data packets to compensate for clock drift. However, single time source neighbour scheme endangers de-synchronisation in the wireless network. In this letter, we propose using multiple time source parents to improve the synchronisation of the the network.
\end{abstract}

KEYWORDS:

Industrial IoT, IEEE 802.15.4-2015, TSCH, Guard Time, Synchronisation, Multiple Time Sources

\section{1 | INTRODUCTION}

Internet Protocol version 6 (IPv6) is considered as the "thin waist" of the Internet. Due to its widespread usage, thousands and thousands constrained devices in terms of computation and memory are connected to the Internet, which enables a new paradigm called the Internet of Things (IoT) ${ }^{1}$. In fact, during the last decade, there has been a great trend in developing and deploying IoT-based technology, with industrial domain ranging from Smart Grid and Factories ${ }^{2}$.

Industrial IoT (IIoT) applications require ultra reliable communication between the IoT nodes and potentially bounded latency ${ }^{3}$. In 2016, the IEEE std 802.15.4-2015 standard was published. Among the Medium Access Control (MAC) protocols standardised in this document, Time Slotted Channel Hopping (TSCH) is the protocol defined to fullfil the industrial requirements ${ }^{4}$. TSCH is a Time Division Multiple Access (TDMA) based protocol, where the nodes must continuously remain time-synchronised by systematically compensating for the clock drifts. For a pair of devices, a transmitter and a receiver, to guarantee a reliable communication, the receiver turns $\mathrm{ON}$ its radio to listen for the wireless medium shortly before the scheduled time of a frame arrival. This offset period between the time a receiver starts actually listening the wireless medium and the expected time of frame arrival is called guard time. This guard time period is defined in the standard to reduce the probability of missed frames due to clock drift. Ultra-high reliability and energy-efficiency is ensured by maintaining good time synchronization. Related work on time synchronization on TSCH networks focuses on a single time-source. In contrast, this letter proposes multiple time sources to guarantee continuous and reliable synchronization among the devices.

\section{2 | TECHNICAL BACKGROUND \& PROBLEM FORMULATION}

IEEE std 802.15.4-2015 TSCH guarantees performance levels similar to the industrial standards, such as ISA ${ }^{5}$, WirelessHART ${ }^{6}$. This section provides the technical background about the TSCH functionality as well as about the re-synchronisation procedures. 


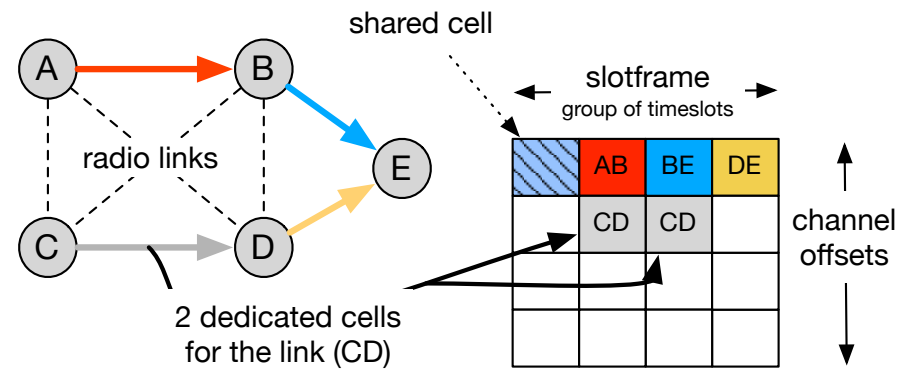

FIGURE 1 A typical TSCH schedule is illustrated for a 5-node topology.

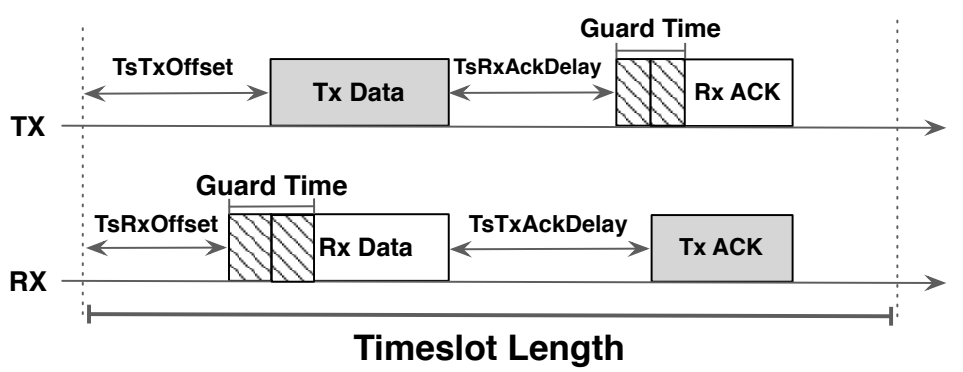

FIGURE 2 The mechanics of a TSCH sender (top) and receiver (bottom): The sender sends its data packet after TxOffset, while the receiver wakes up before the scheduled transmission to account for clock drifts.

\section{1 | IEEE std 802.15.4-2015 TSCH Overview}

To provide high level of network reliability, low end-to-end latency and energy-efficient operation, TSCH operates over a schedule that is based on Frequency Division Multiple Access (FDMA) and TDMA modes of operations. In fact, the TSCH schedule is a matrix of channel offsets and timeslots that represents the cells, and that continuously repeats over time. A group of timeslots construct the slotframe. To each pair of nodes (radio link), set of cells (timeslot and channel offset) are allocated to indicate when the transmissions will take place. As a result, the packet transmissions and the receptions are $a$ priori and strictly scheduled. The cells are either contention-free, dedicated cells for data place that are in unicast mode, or contention-based, shared cells for control plane that are in broadcast mode. In Fig. 1] a typical TSCH schedule is depicted.

In TSCH, the time is organized into timeslots of equal size, typically of $10 \mathrm{~ms}$. The $10 \mathrm{~ms}$ of a timeslot is sufficient for a node to transmit a packet and to receive an acknowledgement, see Fig. 2

\section{2 | Guard Time}

In embedded systems, timing events are controlled by crystals which oscillate at a certain frequency. Due to production spread, i.e. precision differences and structural imperfections of the hardware, this frequency slightly differs from crystal to crystal. In addition, the resonating frequency also depends on the operating temperature ${ }^{7}$. As a result, the clocks of different TSCH nodes beat at a slightly different frequency, resulting in clock drift, which requires periodic synchronisation. TSCH incorporates the guard time as a way to maintain the communication between synchronisation events.

Fig. 2 2illustrates the mechanics of a TSCH packet transmission. A sender node with data to transmit, waits for TxOffset before transmitting its data packet. The receiver node wakes up some time in advance, in order to protect the communication from some synchronisation error. Indeed, the clock drift can be either positive or negative. To account for both cases, the receiver node turns its radio $\mathrm{ON}$ before the expected transmission for up to $\tau$, unless a preamble is successfully received earlier. This time interval corresponds to the guard time and is equally spaced around the end of the TxOffset. 

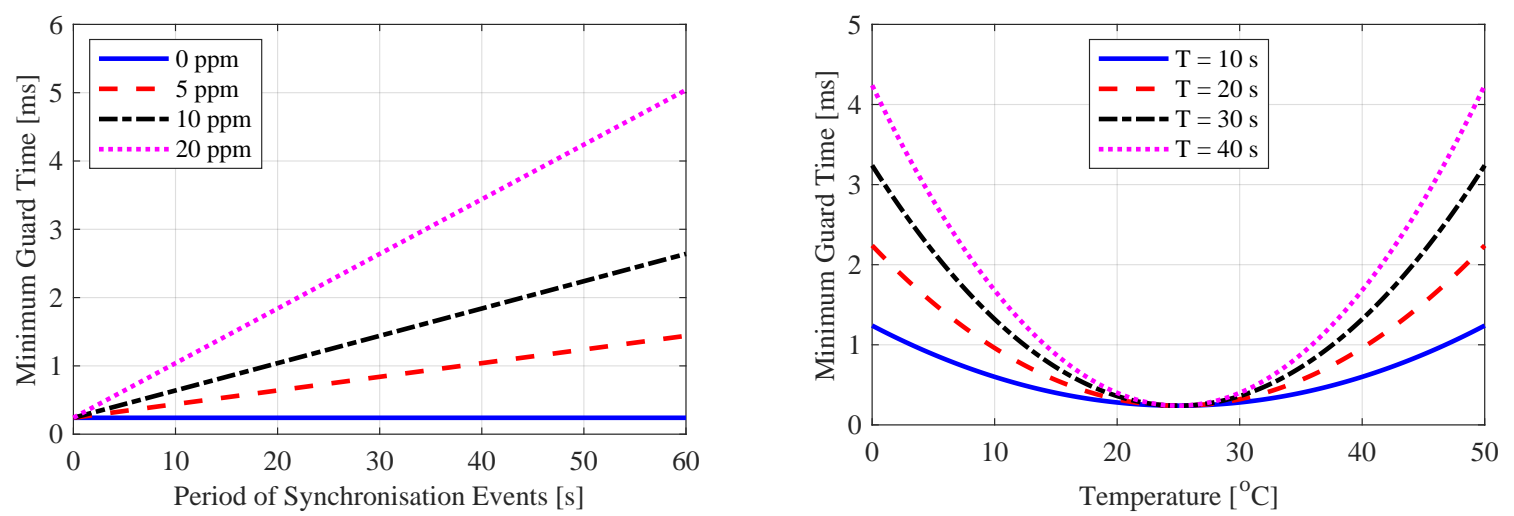

FIGURE 3 The minimum guard time for various periods of synchronisation (left) and temperature differences (right).

\section{3 | Time Synchronisation}

As previously mentioned, the clocks of wireless devices may drift apart relatively to their neighbours' due to production spread or due to temperature changes. Once a node joins a TSCH network, it is assigned with a time source neighbour to which it must remain synchronised constantly by canceling the clock drift. According to the IEEE std 802.15.4-2015 TSCH, there are two ways for a node to re-synchronise to its time source neighbour, the frame-based and the ACK-based synchronisation. More specifically, at each re-synchronisation, the receiver calculates the relative de-synchronisation time, called off set. Then, in frame-based synchronisation, this of $f$ set is applied at the receiver's timing, while in ACK-based synchronisation, it is handled at the transmitter's timing. Note that both control packets such as Enhanced Beacon (EB) as well as data packet packets may be employed to first calculate the potential clock drifts and then to re-synchronise the clocks ${ }^{8}$.

Fig. 3 (left) shows the minimum guard time $\left(\tau_{m}\right)$ for various periods of synchronisation events $(T)$ and for different clock errors $\left(e_{f}\right)$. The minimum guard time increases linearly with the period of packet transmissions that act as synchronisation events. Hence, the default guard time of the TSCH Contiki implementation ${ }^{9}, 2200 \mu \mathrm{s}$, requires at least one transmission per 20 seconds, assuming a clock error of $e_{f}= \pm 20 \mathrm{ppm}$.

In addition to production spread, clock drifts are also temperature-dependent. In particular, the low-cost crystal oscillators, typically used in sensor nodes, are manufactured so that their frequency dependence on temperature $(K)$ is quadratic with the maximum around $K_{0}=25^{\circ} \mathrm{C}$ (see for example the FC-135 used in SensorTag and SPES- $2^{10}$ ). As a result, a typical crystal oscillator operates close to its nominal frequency at room temperature, but slows down at outdoor temperatures. These behaviour is characterised by a parabolic coefficient $B$. The temperature-dependent clock drift $\left(e_{K}\right)$ is given by:

$$
e_{K}=B\left(K-K_{0}\right)^{2} .
$$

Assuming a point-to-point link where the sender operates at $K_{0}$ and the receiver operates at $K$, the minimum guard time required to avoid loss of synchronisation $\left(\tau_{K}\right)$ is given by:

$$
\tau_{K}=2 T\left(\frac{1}{1-e_{K}}-1\right)+2 \tau_{p}
$$

Fig 3 (right) shows the minimum guard time $\left(\tau_{K}\right)$ for various periods of synchronisation $(T)$ and for different temperatures $(K)$. The numerical results consider the parabolic coefficient value of $\mathrm{FC}-135, B=0.04 \mathrm{ppm} /{ }^{\circ} \mathrm{C}^{2}$. The results suggest that the guard time of the Contiki implementation requires at least one transmission per 20 seconds at low temperatures (e.g., $0^{\circ} \mathrm{C}$ ).

\section{3 | CHALLENGES OF SINGLE TIME SOURCE}

Let us consider a 6TiSCH network, where TSCH is the MAC protocol and IPv6 Routing Protocol for Low-Power and Lossy Networks (RPL) is the routing protocol ${ }^{11}$. RPL manages the network by constructing a Direction-Oriented Directed Acyclic Graph (DODAG) using a distance-vector algorithm whereby each node selects one or more routing parent(s), acting as relay nodes toward the root (i.e., the border router) according to the employed objective function ${ }^{12}$. In 6TiSCH based network, this DODAG structure is used for the synchronization where a node's routing parent acts as the time source neighbor as well. 


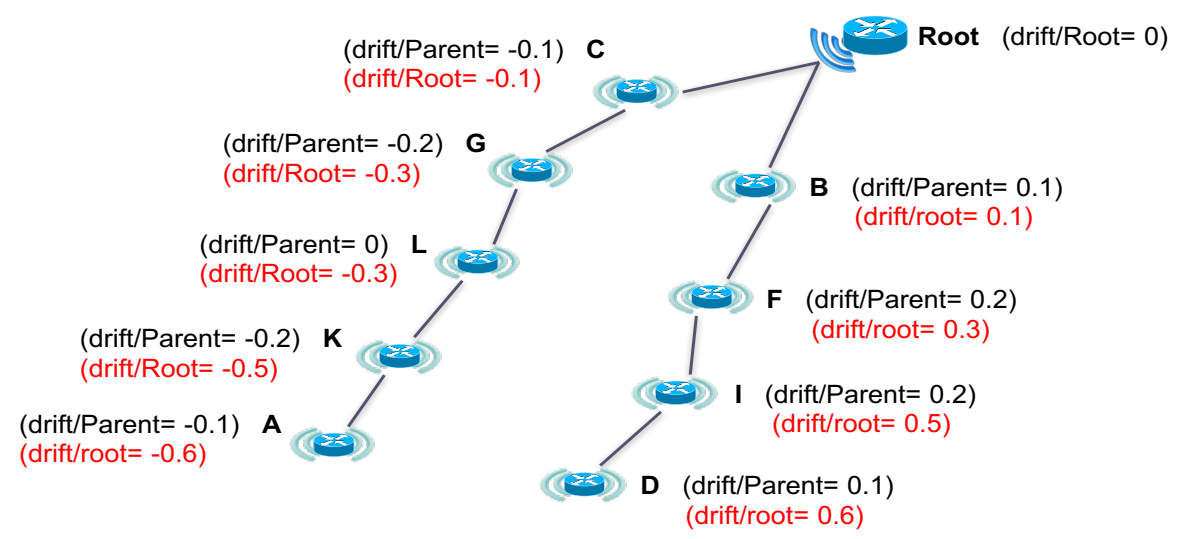

FIGURE 4 In this paper, we consider a fork topology as shown in the figure. In this example, the relative drift to the parent node is shown in black and the relative drift to the root node is shown in red. It can be observed that in the worst case scenario, whereby the time drifts do not cancel out, the relative drift to the root increases with the network depth.

\subsection{De-synchronization from the Root}

The problem raises in multi-hop 6TiSCH network where each node has only one time source parent. More specifically, in such multi-hop wireless network, the de-synchronization clock drift adds up with the number of hops to the root. Then, if accurate and efficient re-synchronization procedure will not take place, a "synchronization swing" may happen which practically means a de-synchronization of nodes deeper in the $6 \mathrm{TiSCH}$ network ${ }^{8}$. In Fig. 4 a synchronization swing in the canonical case of a linear network of 4 nodes is depicted.

\section{2 | Routing Parent Changes}

In 6TiSCH networks, it is common for a node to change its preferred parent, for instance, due to link quality changes or load balancing purposes. Taking as an example the topology depicted in Fig. 4. node $\mathrm{G}$ would change its preferred parent from $\mathrm{C}$ to B if it realizes that the link quality to B is better than to C. In that case, the deeper a node is in the network the harder or even impossible would be for it to switch the routing parent. For instance, node A will not be able to switch to any of I and D routing parents, since their clocks are drifted apart and are de-synchronized.

\section{4 | MULTI-SOURCE VS SINGLE SOURCE}

In this letter, we propose to employ multiple time source neighbours instead of one to achieve better precision. According to theRPL protocol, each node has a list of parents, one is selected as the preferred parent to relay the traffic. We propose that a node will proceed with the re-synchronisation by considering multiple routing parents as time source. By averaging the drifts from multiple parents will reduce the probability of being de-synchronised from the root node as well as will allow for nodes deeper in the network to changes routing parents smoothly.

\section{1 | Routing Parent Changes}

In this section, we present a numerical experiment to demonstrate the benefit of multiple time-sources in the scenario of routing parent changes. Consider the scenario that node $\mathrm{A}$ is using node $\mathrm{K}$ as its original parent node. Assuming that $e_{f}$ is the maximum drift to the parent node and they re-synchronise once every $T$ seconds, the maximum synchronisation error from the original parent node $\left(E_{o}^{1}\right)$ is given by:

$$
E_{o}^{1}=e_{f} T .
$$



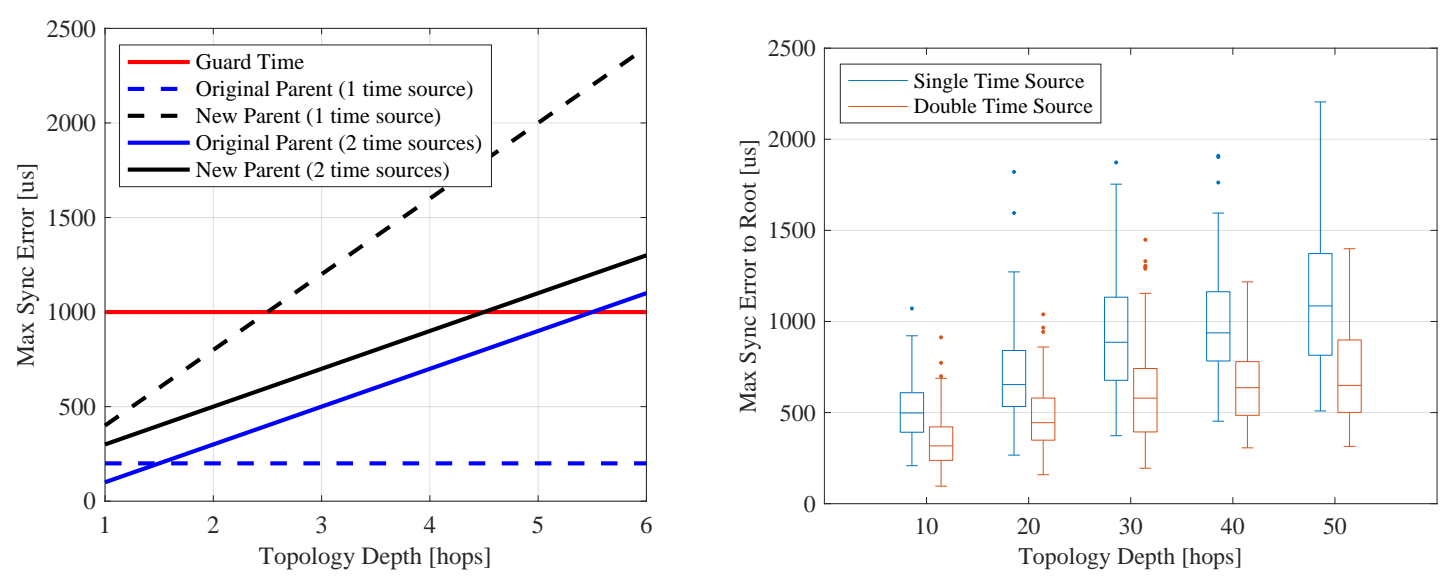

FIGURE 5 The benefits of employing two time sources for the cases of parent changes (left) and de-synchronisation from the root node (right).

Let us consider now that A needs to switch to parent D. In the worst case scenario, whereby one chain at maximum positive drift and other chain at maximum negative drift, the maximum synchronisation error of A from the new parent node $\left(E_{n}^{1}\right)$ is given by

$$
E_{n}^{1}=2 N e_{f} T,
$$

where $N$ is the depth of the network in number of hops. Let us now reconsider the same scenario, but in this case A uses two time sources, namely both $\mathrm{K}$ and $\mathrm{D}$, averaging the respective time drifts. In the case of two time sources, the maximum synchronisation error from the original parent node $\left(E_{o}^{2}\right)$ is given by:

$$
E_{o}^{2}=\frac{2 N e_{f}-e_{f}}{2} T \text {. }
$$

Similarly, the maximum synchronisation error from the new parent node $\left(E_{n}^{2}\right)$ is given by:

$$
E_{o}^{2}=\frac{2 N e_{f}+e_{f}}{2} T \text {. }
$$

Figure 5 (left) plots the maximum synchronisation error to the parent node in these four cases for $e_{f}=20 \mathrm{ppm}, T=10$ seconds and for various topology depths $(N)$. The results suggest that with two time sources the network is able to maintain synchronisation with smaller guard times, since the maximum synchronisation error is contained. This results in significant energy saving since the nodes spend are less time in radio active mode $\mathrm{I}^{13}$.

\section{2 | De-synchronisation from the Root}

Next, we present a numerical experiment to demonstrate the benefit of multiple time-sources in maintain synchronisation with the root node. In this experiment, we consider that each node has a random drift, $e_{i j}$ from a uniform distribution with maximum value $\pm e_{f}$, where $j=o$ in the case of the original parent and $j=n$ in the case of the alternative parent. Similarly, each node re-synchronises with its time source once every $T$ seconds. In case of a single time source, the maximum synchronisation error to the root node is given by

$$
E_{i}^{1}=E_{i-1}^{1}+e_{i o} T .
$$

In case of two time sources, the maximum synchronisation error to the root node is given by

$$
E_{i}^{2}=E_{i-1}^{2}+\frac{e_{i o}+e_{i n}}{2} T
$$

Figure 5 (right) plots the maximum synchronisation error to the root node for $e_{f}=20 \mathrm{ppm}, T=10$ seconds and for various topology depths $(N)$. In particular, the process is repeated 100 times and we plot box plots of the maximum absolute synchronisation error in the network, i.e. $\max \left(E_{j}^{1}\right)$ and $\max \left(E_{j}^{2}\right)$ respectively. The results demonstrate the reduction of the maximum synchronisation to the root node when employing two time sources. 


\section{5 | CONCLUSIONS}

TSCH is one of the medium access control modes in IEEE std 802.15.4-2015 standard. It combines the fundamental TDMA and FDMA protocols. Therefore, under TSCH network, the devices must remain constantly synchronised. To compensate the potential clock drift due to production spread or due to temperature changes, the nodes exchange data packets with their time source neighbour. In case of low traffic application, the nodes use the control packets that are sent periodically such as the EB.

However, when only one time source neighbour is employed in the network per device, then the nodes that are located deeper in the network are endangered to be de-synchronised. Furthermore, for these nodes it would be harder to find another parent in case they need to change their routing parent, for instance due to link quality variation. In this letter, we studied de-synchronisation issues in 6TiSCH networks, and proposed multi-neighbour time source to avoid de-synchronisation. Indeed, our theoretical analysis demonstrates that two time sources reduce the maximum de-synchronisation to the root node as well as the maximum synchronisation error is contained and, thus, two nodes are capable to maintain synchronisation with smaller guard time values.

In future work we plan to implement the proposed algorithm on real-world hardware, such as OpenMotes. Furthermore, we plan to investigate the performance of the algorithm on more general topologies and on more than two time source neighbors.

\section{References}

1. Kritsis K, Papadopoulos GZ, Gallais A, Chatzimisios P, Theoleyre F. A Tutorial on Performance Evaluation and Validation Methodology for Low-Power and Lossy Networks. IEEE Communications Surveys and Tutorials 2018; 20: 1799-1825.

2. Papadopoulos GZ, Thubert P, Theoleyre F, Bernardos C. RAW Use Cases. IETF RAW WG draft-bernardos-raw-use-cases01 [work-in-progress]; 2019.

3. Koutsiamanis RA, Papadopoulos GZ, Fafoutis X, Fiore JMD, Thubert P, Montavont N. From Best-Effort to Deterministic Packet Delivery for Wireless Industrial IoT Networks. IEEE Transactions on Industrial Informatics 2018; 14: 4468-4480.

4. IEEE Standard for Low-Rate Wireless Personal Area Networks (LR-WPANs). IEEE Std 802.15.4-2015 (Revision of IEEE Std 802.15.4-2011); 2016.

5. ISA-100.11a-2011: . Wireless Systems for Industrial Automation:Process Control and Related Applications. International Society of Automation (ISA) Std. May 2011; 1.

6. WirelessHART Specification 75: TDMA Data-Link Layer. HART Communication Foundation Std., Rev. 1.1, 2008; HCF SPEC-75.

7. Elsts A, Fafoutis X, Duquennoy S, Oikonomou G, Piechocki R, Craddock I. Temperature-resilient time synchronization for the internet of things. IEEE Transactions on Industrial Informatics 2017; 14(5): 2241-2250.

8. Chang T, Watteyne T, Pister K, Wang Q. Adaptive Synchronization in Multi-hop TSCH Networks. Computer Networks 2015; 76: 165-176.

9. Duquennoy S, Elsts A, Nahas BA, Oikonomou G. TSCH and 6TiSCH for Contiki: Challenges, Design and Evaluation. In: 13th Int. Conf. on Distributed Comput. in Sensor Syst. (DCOSS). ; 2017.

10. Fafoutis X, Elsts A, Vafeas A, Oikonomou G, Piechocki R. Demo: SPES-2 - A Sensing Platform for Maintenance-Free Residential Monitoring. In: Proceedings of the EWSN. ; 2017.

11. Winter T, Thubert P, Brandt A, et al. RPL: IPv6 Routing Protocol for Low-Power and Lossy Networks. IETF RFC 6550; 2012.

12. Gnawali O, Levis P. The Minimum Rank with Hysteresis Objective Function. RFC 6719 (Proposed Standard); 2012.

13. Papadopoulos GZ, Mavromatis A, Fafoutis X, et al. Guard Time Optimisation and Adaptation for Energy Efficient Multi-hop TSCH Networks. In: Proceedings of the IEEE 3rd World Forum on Internet of Things (WF-IoT). ; 2016: 301-306. 